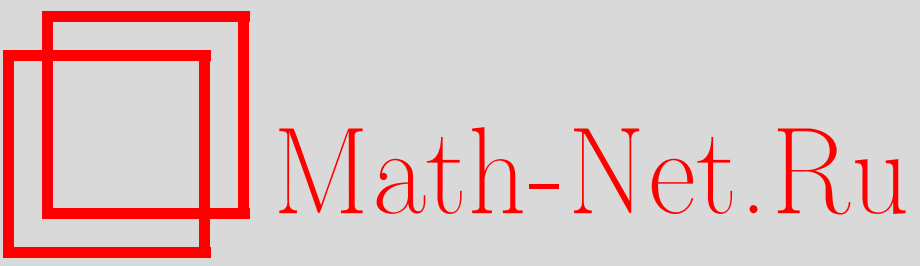

М. А. Урусов, Использование разделяющих моментов для доказательства сингулярности гауссовских мер, УМH, 2003, том 58, выпуск 4, 163-164

DOI: https://doi.org/10.4213/rm656

Использование Общероссийского математического портала Math-Net.Ru подразумевает, что вы прочитали и согласны с пользовательским соглашением

http://www . mathnet.ru/rus/agreement

Параметры загрузки:

IP : 54.147 .182 .235

26 апреля 2023 г., 15:33:13 


\title{
ИСПОЛЬЗОВАНИЕ РАЗДЕЛЯЮЩИХ МОМЕНТОВ ДЛЯ ДОКАЗАТЕЛЬСТВА СИНГУЛЯРНОСТИ ГАУССОВСКИХ МЕР
}

\author{
M. А. УРуСОВ
}

1. Следующий результат известен (см., например, [1; гл. VIII, замечание после теоремы (2.2)]).

Teоpema 1. Пусть $\varphi \in C([0, \infty)), \varphi(0)=0$, где $C([0, \infty))$ - пространство непрерывных функиий $[0, \infty) \rightarrow \mathbb{R}$. Обозначим через $\mathrm{P}$ винеровскую меру на $\mathscr{B}(C([0, \infty)))$, через $\widetilde{\mathrm{P}}$ - распределение процесса $\left(B_{t}+\varphi(t)\right)_{t \in[0, \infty)}$, әде $\left(B_{t}\right)_{t \in[0, \infty)}$ - стандартное броуновское движение. Тогда справедливы следующие утверждения.

(i) Если ч абсолютно непрерывна на каждом конечном отрезке $u \int_{0}^{\infty}\left(\varphi^{\prime}(s)\right)^{2} d s<\infty$, mo $\widetilde{\mathrm{P}} \sim \mathrm{P}$.

(ii) Если не выполнены условия (i), то $\widetilde{\mathrm{P}} \perp \mathrm{P}$.

В п. 2 мы вводим понятие разделяющего момента, а в п. 3 мы приводим простое доказательство теоремы 1 , использующее разделяющие моменты.

2. Рассмотрим измеримое пространство $(\Omega, \mathscr{F})$ с непрерьвной справа филштрацией $\left(\mathscr{F}_{t}\right)_{t \in[0, \infty)}\left(\mathscr{F}_{t}=\bigcap_{\varepsilon>0} \mathscr{F}_{t+\varepsilon}\right)$. Если $\tau-$ момент остановки, то через $\mathscr{F}_{\tau}$ обозначим $\sigma$-алгебру, определенную по формуле $\mathscr{F}_{\tau}=\left\{A \in \mathscr{F}: A \cap\{\tau \leqslant t\} \in \mathscr{F}_{t}\right.$ для всех $\left.t \in[0, \infty)\right\}$ (в частности, $\mathscr{F}{ }_{\infty}=\mathscr{F}$ ). Пусть Р и $\widetilde{\mathrm{P}}-$ вероятностные меры на $(\Omega, \mathscr{F})$. Как обычно, мы обозначаем через $\mathrm{P}_{\tau}$ (соответственно, $\widetilde{\mathrm{P}}_{\tau}$ ) ограничение $\mathrm{P}\left(\right.$ соответственно, $\widetilde{\mathrm{P}}$ ) на $\mathscr{F}_{\tau}$.

Присоединим к $[0, \infty]$ точку $\delta$ такую, что $\delta>\infty$.

ОПРЕДЕЛЕниЕ 2. Отображение $S: \Omega \rightarrow[0, \infty] \cup\{\delta\}$ назовем расширенным моментом остановки, если $\{S \leqslant t\} \in \mathscr{F}_{t}$ для любого $t \in[0, \infty]$.

ПредЛОЖениЕ 3. (i) Существует расширенный момент остановки $S$ такой, что для любого момента остановки $\tau$

$$
\begin{aligned}
& \widetilde{\mathrm{P}}_{\tau} \sim \mathrm{P}_{\tau} \text { на множестве }\{\tau<S\}, \\
& \widetilde{\mathrm{P}}_{\tau} \perp \mathrm{P}_{\tau} \text { на множестве }\{\tau \geqslant S\} .
\end{aligned}
$$

(ii) Если $S^{\prime}$ - другой расширенный момент остановки с этими свойствами, то $S^{\prime}=S$ P,$\widetilde{\mathrm{P}}-n$. .

ОПРЕДЕЛЕНИЕ 4. Любой расширенный момент остановки, удовлетворяющий (1) и (2) для всех моментов остановки $\tau$, назовем разделяющим моментом для $\mathrm{P} u \widetilde{\mathrm{P}}$.

Понятие разделяющего момента введено в [2], где оно используется для получения критериев локалннй абсолютной непрерывности, абсолютной непрерьвности и сингулярности мер $\mathrm{P}$ и $\widetilde{\mathrm{P}}$ в случаях, когда Р и $\widetilde{\mathrm{P}}$ - распределения процессов Леви, решений СДУ и процессов Бесселя.

3. Прежде чем доказывать теорему 1 , сформулируем некоторые вспомогательные утверждения

Лемма 5. Рассмотрим постановку п. 2. Пусть $S$-разделяющий момент для Р и $\widetilde{\mathrm{P}}$. Тогда

$$
\widetilde{\mathrm{P}}_{0} \perp \mathrm{P}_{0} \Longleftrightarrow S=0 \quad \mathrm{P}, \widetilde{\mathrm{P}}_{-n . \mu .} \Longleftrightarrow S=0 \quad \mathrm{P}-n . \text { } .
$$

Доказательство проводится непосредственно.

Лемма 6. Рассмотрим некоторый стохастический базис $\left(\Omega, \mathscr{F},\left(\mathscr{F}_{t}\right), \mathrm{P}\right)$. Пусть $\varphi \in$ $C([0, \infty)),\left(A_{t}\right)_{t \in[0, \infty)}$ - непрерывный согласованный процесс, имеющий ограниченную вариацию на конечных отрезках, $\tau$ - момент остановки, $\mathrm{P}(\tau>0)>0$. Предположим, что прочесс $\left(\varphi(t \wedge \tau)-A_{t \wedge \tau}\right)_{t \in[0, \infty)}$ является локальным мартингалом. Тогда существует $\varepsilon>0$ такое, что функция $\varphi$ имеет ограниченную вариацию на отрезке $[0, \varepsilon]$. 
Доказательство аналогично доказательству того, что детерминированньй семимартингал имеет ограниченную вариацию на конечных отрезках (см. [3; гл. I, предложение 4.28]).

Лемма 7. В условиях теоремы 1 пусть либо ч не является абсолютно непрерывной функцией на отрезке $[0, t]$ для любого $t>0$, либо $\varphi$ абсолютно непрерывна на $[0, t]$ при достаточно мальх $t>0$, но $\int_{0}^{t}\left(\varphi^{\prime}(s)\right)^{2} d s=\infty$ для любого $t>0$. Тогда $\widetilde{\mathrm{P}} \perp \mathrm{P}$.

ДокАЗАТЕЛЬСтво. Обозначим через $X$ канонический процесс на $C([0, \infty))$, т.е. процесс, определенньй по формуле $X_{t}(\omega)=\omega(t)$. Рассмотрим фильтрацию $\mathscr{F} t=\bigcap_{\varepsilon>0} \sigma\left(X_{s} ; s \in\right.$ $[0, t+\varepsilon])$ и положим $\mathscr{F}=\bigvee_{t \in[0, \infty)} \mathscr{F}_{t} .($ Тогда $\mathscr{F}=\mathscr{B}(C([0, \infty)))$.) Пусть $S-$ разделяющий момент для Р и $\widetilde{\mathrm{P}}$.

Легко видеть, что $\sigma$-алгебра $\mathscr{F}_{0}$ тривиальна по каждой из мер Р и $\widetilde{\mathrm{P}}$. Отсюда и из леммы 5 получаем, что либо $S=0 \mathrm{P}, \widetilde{\mathrm{P}}$-п.н., либо $S>0 \mathrm{P}, \widetilde{\mathrm{P}}$-п.н. Докажем, что второй вариант невозможен.

Предположим противное: $S>0$ Р,$\widetilde{\mathrm{P}}$-п.н., или, что эквивалентно, $\widetilde{\mathrm{P}}_{0} \not \perp \mathrm{P}_{0}$. Поскольку любой момент остановки на винеровской фильтрации п.н. совпадает с предсказуемым моментом, то существуют моменты остановки $\tau^{\prime}$ и $\tau^{\prime \prime}$ такие, что $0<\tau^{\prime}<S$ Р-п.н. и $0<\tau^{\prime \prime}<S$ Р-п.н.

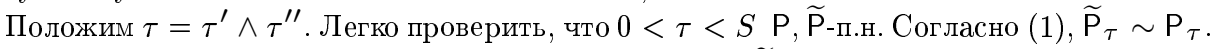

Рассмотрим càdlàg $\left(\mathscr{F}_{t}, \mathrm{P}\right)$-мартингал $Z_{t}=\mathrm{E}_{\mathrm{P}}\left(\frac{d \widetilde{\mathrm{P}}_{\tau}}{d \mathrm{P}_{\tau}} \mid \mathscr{F}_{t}\right), t \in[0, \infty)$. Отметим, что $Z$ является равномерно интегрируемым мартингалом с предельньм значением $Z_{\infty}=\frac{d \widetilde{\mathrm{P}}_{\tau}}{d \mathrm{P}_{\tau}}$. Поскольку $Z_{\infty}>0$ Р-п.н., то процесс $Z$ строго положителен относительно меры Р. Согласно [4; гл. III, § 3c, теорема 2], существует предсказуемый процесс $\beta$ такой, что $\int_{0}^{t} \beta_{s}^{2} d s<\infty$ Р-п.н., $t \in[0, \infty)$, и $Z_{t}=\exp \left\{\int_{0}^{t} \beta_{s} d X_{s}-\frac{1}{2} \int_{0}^{t} \beta_{s}^{2} d s\right\}$ Р-п.н., $t \in[0, \infty)$, где стохастический интеграл понимается относительно меры $\mathrm{P}$.

Введем меру $\mathrm{Q}=Z_{\infty} \cdot \mathrm{P}$. Тогда $\mathrm{Q}_{\tau}=\widetilde{\mathrm{P}}_{\tau}$. C использованием теоремы Гирсанова отсю-

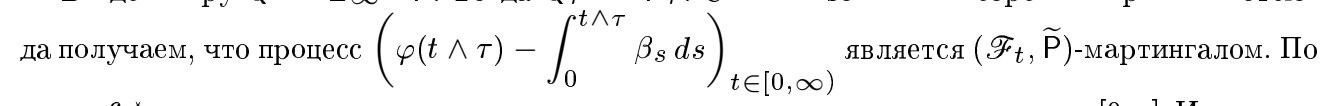
лемме 6 функция $\varphi$ имеет ограниченную вариацию на достаточно малом отрезке $[0, \varepsilon]$. Используя $\widetilde{\mathrm{P}}_{\tau} \sim \mathrm{P}_{\tau}$, отсюда можно получить, что $\mathrm{P}\left(\forall t \in[0, \varepsilon]: \varphi(t \wedge \tau)=\int_{0}^{t \wedge \tau} \beta_{s} d s\right)=1$. Вместе с тем, что $\tau>0$ Р-п.н. и $\int_{0}^{t} \beta_{s}^{2} d s<\infty$ Р-п.н., $t \in[0, \infty)$, это противоречит условиям леммші.

ДокАЗАТЕЛЬСТво тЕОРЕмы 1. Достаточно доказать лишь утверждение (ii). Его нетрудно получить из теоремы Гирсанова и леммы 3.

Автор благодарит А. Н. Ширяева и А. С. Черного за полезные советы и предложения.

\section{СПИСОК ЛИТЕРАТУРЫ}

[1] D. Revuz, M. Yor. Continuous Martingales and Brownian Motion. Berlin: SpringerVerlag, 1994. [2] М. А. Урусов, А. С. Черный // Теория вероятн. и ее примен. 2003. Т. 48. № 2. С. 416-427. [3] ЖК. ЖКакод, А. Н. Ширяев. Предельные теоремы для случайных процессов. М.: Физматлит, 1994. [4] А. Н. Ширяев. Основы стохастической финансовой математики. М.: Фазис, 1998. 